\title{
EDGEWORTH EXPANSIONS OF SOME STATISTICS INCLUDING THE LB-STATISTIC AND V-STATISTIC
}

\author{
Hajime Yamato*, Toshifumi Nomachi** and Koichiro Toda***
}

\begin{abstract}
As an estimator of an estimable parameter, Toda and Yamato (2001) introduced a linear combination $Y_{n}$ of U-statistics which includes V-statistic and LB-statistic. We give the Edgeworth expansions of the standardized $Y_{n}$ statistic with remainder $o\left(n^{-1}\right)$ in case that the kernel is not degenerate. We also give the Edgeworth expansions of the studentized $Y_{n}$ statistic using a jackknife variance estimator with remainder $o\left(n^{-1 / 2}\right)$.
\end{abstract}

Key words and phrases: Edgeworth expansion, linear combination of U-statistics, V-statistic.

\section{Introduction}

Let $\theta(F)$ be an estimable parameter of an unknown distribution $F$ and $g\left(x_{1}, \ldots, x_{k}\right)$ be its symmetric kernel of degree $k(\geq 2)$. In this paper, we assume that the kernel $g$ is not degenerate. Let $X_{1}, \ldots, X_{n}$ be a random sample of size $n$ from the distribution $F$. As an estimator of $\theta(F)$, a linear combination $Y_{n}$ of U-statistics is introduced by Toda and Yamato (2001) as follows: Let $w\left(r_{1}, \ldots, r_{j} ; k\right)$ be a nonnegative and symmetric function of positive integers $r_{1}, \ldots, r_{j}$ such that $j=1, \ldots, k$ and $r_{1}+\cdots+r_{j}=k$, where $k$ is the degree of the kernel $g$ and is fixed. We assume that at least one of $w\left(r_{1}, \ldots, r_{j} ; k\right)$ 's is positive. For $j=1, \ldots, k$, let $g_{(j)}\left(x_{1}, \ldots, x_{j}\right)$ be the kernel given by

$$
\begin{aligned}
& g_{(j)}\left(x_{1}, \ldots, x_{j}\right) \\
& =\frac{1}{d(k, j)} \sum_{r_{1}+\cdots+r_{j}=k}^{+} w\left(r_{1}, \ldots, r_{j} ; k\right) g(\underbrace{x_{1}, \ldots, x_{1}}_{r_{1}}, \ldots, \underbrace{x_{j}, \ldots, x_{j}}_{r_{j}}),
\end{aligned}
$$

where the summation $\sum_{r_{1}+\cdots+r_{j}=k}^{+}$is taken over all positive integers $r_{1}, \ldots, r_{j}$ satisfying $r_{1}+\cdots+r_{j}=k$ with $j$ and $k$ fixed and $d(k, j)=\sum_{r_{1}+\cdots+r_{j}=k}^{+} w\left(r_{1}, \ldots\right.$, $\left.r_{j} ; k\right)$ for $j=1,2, \ldots, k$. Let $U_{n}^{(j)}$ be the U-statistic associated with this kernel $g_{(j)}\left(x_{1}, \ldots, x_{j} ; k\right)$ for $j=1, \ldots, k$. The kernel $g_{(j)}\left(x_{1}, \ldots, x_{j} ; k\right)$ is symmetric because of the symmetry of $w\left(r_{1}, \ldots, r_{j} ; k\right)$. If $d(k, j)$ is equal to zero for some $j$, then the associated $w\left(r_{1}, \ldots, r_{j} ; k\right)$ 's are equal to zero. In this case, we let the

\footnotetext{
Received March 29, 2002. Revised July 23, 2002. Accepted November 19, 2002.

*Department of Mathematics and Computer Science, Kagoshima University, Kagoshima 890-0065, Japan.

**Miyakonojo College of Technology, Miyazaki 885-8567, Japan.

***Graduate School of Science and Engineering, Kagoshima University, Kagoshima 890-0065, Japan.
} 
corresponding statistic $U_{n}^{(j)}$ be zero. The statistic $Y_{n}$ is given by

$$
Y_{n}=\frac{1}{D(n, k)} \sum_{j=1}^{k} d(k, j)\left(\begin{array}{c}
n \\
j
\end{array}\right) U_{n}^{(j)},
$$

where $D(n, k)=\sum_{j=1}^{k} d(k, j)\left(\begin{array}{c}n \\ j\end{array}\right)$. Since $w$ 's are nonnegative and at least one of them is positive, $D(n, k)$ is positive. Note that $U_{n}^{(k)}=U_{n}$ for $w(1, \ldots, 1 ; k)>0$, because $g_{(k)}=g$.

For example, let $w$ be the function given by $w(1,1, \ldots, 1 ; k)=1$ and $w\left(r_{1}, \ldots\right.$, $\left.r_{j} ; k\right)=0$ for positive integers $r_{1}, \ldots, r_{j}$ such that $j=1, \ldots, k-1$ and $r_{1}+\cdots+$ $r_{j}=k$. Then the corresponding statistic $Y_{n}$ is equal to U-statistic $U_{n}$, which is given by

$$
U_{n}=\left(\begin{array}{c}
n \\
j
\end{array}\right)^{-1} \sum_{1 \leq j_{1}<\cdots<j_{k} \leq n} g\left(X_{j_{1}}, \ldots, X_{j_{k}}\right)
$$

where $\sum_{1 \leq j_{1}<\cdots<j_{k} \leq n}$ denotes the summation over all integers $j_{1}, \ldots, j_{k}$ satisfying $1 \leq j_{1}<\cdots<\bar{j}_{k} \leq n$.

Let $w$ be the function given by $w\left(r_{1}, \ldots, r_{j} ; k\right)=1$ for positive integers $r_{1}, \ldots, r_{j}$ such that $j=1, \ldots, k$ and $r_{1}+\cdots+r_{j}=k$. Then the corresponding statistic $Y_{n}$ is equal to the LB-statistic $B_{n}$ given by

$$
B_{n}=\left(\begin{array}{c}
n+k-1 \\
k
\end{array}\right)^{-1} \sum_{r_{1}+\cdots+r_{n}=k} g(\underbrace{X_{1}, \ldots, X_{1}}_{r_{1}}, \ldots, \underbrace{X_{n}, \ldots, X_{n}}_{r_{n}}),
$$

where $\sum_{r_{1}+\cdots+r_{n}=k}$ denotes the summation over all non-negative integers $r_{1}, \ldots$, $r_{n}$ satisfying $r_{1}+\cdots+r_{n}=k$. (The term LB-statistic is the abbreviation for the limit of the Bayes estimate and was introduced in Nomachi and Yamato (2001). For the limit of the Bayes estimate of $\theta$, see Yamato $(1977 \mathrm{a}, \mathrm{b})$.)

Let $w$ be the function given by $w\left(r_{1}, \ldots, r_{j} ; k\right)=k ! /\left(r_{1} ! \cdots r_{j} !\right)$ for positive integers $r_{1}, \ldots, r_{j}$ such that $j=1, \ldots, k$ and $r_{1}+\cdots+r_{j}=k$. Then the corresponding statistic $Y_{n}$ is equal to the $\mathrm{V}$-statistic $V_{n}$ given by

$$
V_{n}=\frac{1}{n^{k}} \sum_{j_{1}=1}^{n} \cdots \sum_{j_{k}=1}^{n} g\left(X_{j_{1}}, \ldots, X_{j_{k}}\right)
$$

(See Toda and Yamato (2001).)

Let $w$ be the function given by $w\left(r_{1}, \ldots, r_{j} ; k\right)=k ! /\left(r_{1} \cdots r_{j}\right)$ for positive integers $r_{1}, \ldots, r_{j}$ such that $j=1, \ldots, k$ and $r_{1}+\cdots+r_{j}=k$. Then, for the case of $k=3$, the corresponding statistic $Y_{n}$ for the third central moment of the distribution $F$ is given by

$$
S_{n}=\frac{n}{n^{2}+1} \sum_{i=1}^{n}\left(X_{i}-\bar{X}\right)^{3}
$$


where $\bar{X}$ is the sample mean of $X_{1}, \ldots, X_{n}$ (see Nomachi et al. (2002)).

In Section 2, we give an approximation of $Y_{n}$ in order to derive its Edgeworth expansions in the following sections. For U-statistics, the Edgeworth expansions of its standardized statistics are studies by Callaert et al. (1980), Bickel et al. (1986), and Maesono (1987). Lai and Wang (1993) introduces asymptotic Ustatistic and gives its Edgeworth expansion. In Section 3, with remainder $o\left(n^{-1}\right)$, we derive the Edgeworth expansion of the standardized $Y_{n}$ statistic using the result of Lai and Wang (1993). We also derive it with remainder $o\left(n^{-1 / 2}\right)$.

For the practical inference of parameter $\theta$, it is useful to get the Edgeworth expansion of the studentized Y-statistic. About a linear combination of U-statistics, $L_{n}$, of Sen (1977), Maesono (1996) gives its Edgeworth expansion for the studentized L-statistic with remainder $o\left(n^{-1}\right)$, using a jackknife variance estimator. While two statistics $Y_{n}$ and $L_{n}$ are both linear combination of U-statistics, $Y_{n}$ is different from $L_{n}$ in the mean that the weight function $w$ determines $Y_{n}$ as an estimator of $\theta$. In Section 4, we derive the Edgeworth expansion of the studentized Y-statistic using a jackknife variance estimator, with remainder $o\left(n^{-1 / 2}\right)$. We will discuss the Edgeworth expansion with remainder $o\left(n^{-1}\right)$ in a future paper. In Section 5, we give examples of Edgeworth expansions of the Y-statistic.

\section{Approximation of $\mathrm{Y}$-statistic}

For the kernel $g_{(j)}\left(x_{1}, \ldots, x_{j}\right)(j=1, \ldots, k)$, we put

$$
\theta_{j}=E g_{(j)}\left(X_{1}, \ldots, X_{j}\right),
$$

and

$$
\psi_{(j), c}\left(x_{1}, \ldots, x_{c}\right)=E\left[g_{(j)}\left(X_{1}, \ldots, X_{j}\right) \mid X_{1}=x_{1}, \ldots, X_{c}=x_{c}\right], \quad c=1, \ldots, j .
$$

For $j=1, \ldots, k$ and $c=2,3, \ldots, k$, we put

$$
\begin{aligned}
& g_{(j)}^{(1)}\left(x_{1}\right)=\psi_{(j), 1}\left(x_{1}\right)-\theta_{j}, \\
& g_{(j)}^{(c)}\left(x_{1}, \ldots, x_{c}\right)=\psi_{(j), c}\left(x_{1}, \ldots, x_{c}\right)-\sum_{i=1}^{c-1} \sum_{1 \leq l_{1}<\cdots<l_{i} \leq c} g_{(j)}^{(i)}\left(x_{l_{1}}, \ldots, x_{l_{i}}\right)-\theta_{j} .
\end{aligned}
$$

Let $H_{(j), n}^{(c)}$ be the U-statistic associated with the kernel $g_{(j)}^{(c)}$, that is,

$$
H_{(j), n}^{(c)}=\left(\begin{array}{c}
n \\
c
\end{array}\right)^{-1} \sum_{1 \leq l_{1}<\cdots<l_{c} \leq n} g_{(j)}^{(c)}\left(X_{l_{1}}, \ldots, X_{l_{c}}\right) .
$$

Then, the U-statistic $U_{n}^{(j)}$ associated with the kernel $g_{(j)}$ can be written as follows,

$$
U_{n}^{(j)}=\theta_{j}+\sum_{c=1}^{j}\left(\begin{array}{l}
j \\
c
\end{array}\right) H_{(j), n}^{(c)} .
$$

This form is known as H-decomposition in the context of U-statistics, because it is due to Hoeffding (see, for example, Lee (1990), p. 26). We note that if 
$d(k, k)=w(1, \ldots, 1 ; k)>0$ then we have $g_{(k)}\left(x_{1}, \ldots, x_{k}\right)=g\left(x_{1}, \ldots, x_{k}\right)$ and so $\theta_{k}=\theta$. Therefore, we shall delete the sub $(k)$. For example, we shall use $g$ and $\theta$ instead of $g_{(k)}$ and $\theta_{k}$, respectively. We shall also abbreviate $\psi_{c}$ and $H_{n}^{(c)}$ instead of $\psi_{(k), c}$ and $H_{(k), n}^{(c)}$, respectively for $c=1,2, \ldots, k$.

In the following, we assume $d(k, k)>0$. Then, there exists a constant $\delta_{k}(\geq 0)$ such that

and

$$
\frac{d(k, k)}{D(n, k)}\left(\begin{array}{l}
n \\
k
\end{array}\right)=1-\frac{\delta_{k}}{n}+O\left(\frac{1}{n^{2}}\right)
$$

Therefore, it follows that

$$
\frac{d(k, k-1)}{D(n, k)}\left(\begin{array}{c}
n \\
k-1
\end{array}\right)=\frac{\delta_{k}}{n}+O\left(\frac{1}{n^{2}}\right) \text {. }
$$

$$
\sum_{j=1}^{k-2} \frac{d(k, j)}{D(n, k)}\left(\begin{array}{l}
n \\
j
\end{array}\right)=O\left(\frac{1}{n^{2}}\right) .
$$

For the U-statistic $U_{n}, d(k, k)\left(\begin{array}{l}n \\ k\end{array}\right) / D(n, k)=1$ and $\delta_{k}=0$. For the V-statistic $V_{n}$ and the S-statistic $S_{n}, \delta_{k}=k(k-1) / 2$. For the LB-statistic $B_{n}, \delta_{k}=k(k-1)$ (see Nomachi et al. (2002)).

Proposition 2.1. We suppose that $d(k, k)>0$ and

$$
E\left[\left|g\left(X_{j_{1}}, \ldots, X_{j_{k}}\right)\right|^{2}\right]<\infty \text { for } 1 \leq j_{1} \leq \cdots \leq j_{k} \leq k .
$$

Then we have

$$
\begin{aligned}
\sqrt{n}\left(Y_{n}-\theta\right)= & k\left(1-\frac{\delta_{k}}{n}\right) \sqrt{n} H_{n}^{(1)}+\left(\begin{array}{c}
k \\
2
\end{array}\right) \sqrt{n} H_{n}^{(2)}+\left(\begin{array}{l}
k \\
3
\end{array}\right) \sqrt{n} H_{n}^{(3)} \\
& +\frac{(k-1) \delta_{k}}{\sqrt{n}} H_{(k-1), n}^{(1)}+\frac{\delta_{k}}{\sqrt{n}}\left(\theta_{k-1}-\theta\right)+R_{n},
\end{aligned}
$$

where $E\left|R_{n}\right|^{2}=O\left(n^{-3}\right)$.

Proof. We put

$$
\sqrt{n}\left(Y_{n}-\theta\right)=\eta_{1}+\eta_{2}+\eta_{3}
$$

where

and

$$
\begin{aligned}
& \eta_{1}=\frac{d(k, k)}{D(n, k)}\left(\begin{array}{l}
n \\
k
\end{array}\right) \sqrt{n}\left(U_{n}-\theta\right), \\
& \eta_{2}=\frac{d(k, k-1)}{D(n, k)}\left(\begin{array}{c}
n \\
k-1
\end{array}\right) \sqrt{n}\left(U_{n}^{(k-1)}-\theta\right),
\end{aligned}
$$

$$
\eta_{3}=\sum_{j=1}^{k-2} \frac{d(k, j)}{D(n, k)}\left(\begin{array}{c}
n \\
j
\end{array}\right) \sqrt{n}\left(U_{n}^{(j)}-\theta\right) .
$$


By Minkowski's inequality and the relation immediately after (2.3), we have

$$
\begin{aligned}
\left(E\left|\eta_{3}\right|^{2}\right)^{1 / 2} & \leq \sum_{j=1}^{k-2} \frac{d(k, j)}{D(n, k)}\left(\begin{array}{c}
n \\
j
\end{array}\right) n^{1 / 2}\left[\left(E\left|U_{n}^{(j)}-\theta_{j}\right|^{2}\right)^{1 / 2}+\left|\theta_{j}-\theta\right|\right] \\
& =O\left(n^{-3 / 2}\right)
\end{aligned}
$$

(see e.g. Lee (1990), p. 120). Hence we have

$$
E\left|\eta_{3}\right|^{2}=O\left(n^{-3}\right)
$$

Using the relation $E\left|H_{(k-1), n}^{(c)}\right|^{2}=O\left(n^{-c}\right), c=1, \ldots, k-1$ (see Serfling (1980), p. 185) and (2.3), we have

$$
\begin{aligned}
\eta_{2} & =\left(\frac{\delta_{k}}{n}+O\left(\frac{1}{n^{2}}\right)\right)\left[\sqrt{n}\left[\sum_{c=1}^{k-1}\left(\begin{array}{c}
k-1 \\
c
\end{array}\right) H_{(k-1), n}^{(c)}\right]+\sqrt{n}\left(\theta_{k-1}-\theta\right)\right] \\
& =\delta_{k}\left[\frac{k-1}{\sqrt{n}} H_{(k-1), n}^{(1)}+\frac{1}{\sqrt{n}}\left(\theta_{k-1}-\theta\right)\right]+R_{2, n},
\end{aligned}
$$

where $E\left|R_{2, n}\right|^{2}=O\left(n^{-3}\right)$. From (2.1) and (2.2), we have

$$
\eta_{1}=\left(1-\frac{\delta_{k}}{n}+O\left(\frac{1}{n^{2}}\right)\right) \sqrt{n}\left[\sum_{c=1}^{k}\left(\begin{array}{l}
k \\
c
\end{array}\right) H_{n}^{(c)}\right]
$$

Since $E\left|H_{n}^{(c)}\right|^{2}=O\left(n^{-c}\right), c=1, \ldots, k$, we have

$$
\eta_{1}=k\left(1-\frac{\delta_{k}}{n}\right) \sqrt{n} H_{n}^{(1)}+\left(\begin{array}{l}
k \\
2
\end{array}\right) \sqrt{n} H_{n}^{(2)}+\left(\begin{array}{l}
k \\
3
\end{array}\right) \sqrt{n} H_{n}^{(3)}+R_{3, n},
$$

where $E\left|R_{3, n}\right|^{2}=O\left(n^{-3}\right)$. Applying (2.7), (2.8) and (2.9) to (2.6), we get (2.5).

We put

$$
Y_{n}^{*}=k\left(1-\frac{\delta_{k}}{n}\right) \sqrt{n} H_{n}^{(1)}+\left(\begin{array}{l}
k \\
2
\end{array}\right) \sqrt{n} H_{n}^{(2)}+\left(\begin{array}{l}
k \\
3
\end{array}\right) \sqrt{n} H_{n}^{(3)}+\frac{(k-1) \delta_{k}}{\sqrt{n}} H_{(k-1), n}^{(1)}
$$

and

$$
\mu_{k}=\delta_{k}\left(\theta_{k-1}-\theta\right)
$$

For any random variables $W$ and $\Delta$, it holds that

$$
\sup _{x}|P(W+\Delta \leq x)-P(W \leq x)| \leq 4(E|W \Delta|+E|\Delta|)
$$

(see Shorack (2000), p. 261). Applying (2.10) to (2.5), we get the following. 
Proposition 2.2. Under the conditions $d(k, k)>0$ and $E\left[\mid g\left(X_{j_{1}}, \ldots\right.\right.$, $\left.\left.X_{j_{k}}\right)\left.\right|^{2}\right]<\infty$ for $1 \leq j_{1} \leq \cdots \leq j_{k} \leq k$, we have

$$
\sup _{-\infty<x<\infty}\left|P\left(\frac{\sqrt{n}}{\sigma}\left(Y_{n}-\theta\right) \leq x\right)-P\left(\frac{1}{\sigma}\left(Y_{n}^{*}+\frac{\mu_{k}}{\sqrt{n}}\right) \leq x\right)\right|=O\left(n^{-3 / 2}\right),
$$

where $\sigma=k \sigma_{1}$ and $\sigma_{1}^{2}=\operatorname{Var}\left[\psi_{1}(X)\right]>0$.

We can write

$$
\sqrt{n} H_{n}^{(2)}=\frac{2}{n^{3 / 2}} \sum_{1 \leq i<j \leq n} g^{(2)}\left(X_{i}, X_{j}\right)+\frac{1}{\sqrt{n}} H_{n}^{(2)}
$$

and have $E\left[\left|H_{n}^{(2)}\right| / \sqrt{n}\right]^{2}=O\left(n^{-3}\right)$. We can also write

$$
\sqrt{n} H_{n}^{(3)}=\frac{6}{n^{5 / 2}} \sum_{1 \leq i<j<l \leq n} g^{(3)}\left(X_{i}, X_{j}, X_{l}\right)+\frac{3 n-2}{n^{3 / 2}} H_{n}^{(3)}
$$

and have $E\left[\left|(3 n-2) H_{n}^{(3)}\right| / n^{3 / 2}\right]^{2}=O\left(n^{-4}\right)$. We put

$$
\begin{aligned}
Y_{n}^{* *}= & k\left(1-\frac{\delta_{k}}{n}\right) \frac{1}{n^{1 / 2}} \sum_{i=1}^{n} g^{(1)}\left(X_{i}\right) \\
& +(k-1) \delta_{k} \frac{1}{n^{3 / 2}} \sum_{i=1}^{n} g_{(k-1)}^{(1)}\left(X_{i}\right) \\
& +k(k-1) \frac{1}{n^{3 / 2}} \sum_{1 \leq i<j \leq n} g^{(2)}\left(X_{i}, X_{j}\right) \\
& +k(k-1)(k-2) \frac{1}{n^{5 / 2}} \sum_{1 \leq i<j<l \leq n} g^{(3)}\left(X_{i}, X_{j}, X_{l}\right) .
\end{aligned}
$$

Applying (2.10) to the above, we get the following.

Corollary 2.3. We have

$$
\sqrt{n}\left(Y_{n}-\theta\right)=Y_{n}^{* *}+\frac{\mu_{k}}{\sqrt{n}}+R_{n}^{\prime}, \quad E\left|R_{n}^{\prime}\right|^{2}=O\left(n^{-3}\right)
$$

and

$$
\sup _{-\infty<x<\infty}\left|P\left(\frac{\sqrt{n}}{\sigma}\left(Y_{n}-\theta\right) \leq x\right)-P\left(\frac{1}{\sigma}\left(Y_{n}^{* *}+\frac{\mu_{k}}{\sqrt{n}}\right) \leq x\right)\right|=O\left(n^{-3 / 2}\right) .
$$




\section{Expansion}

We put as follows:

$$
\begin{aligned}
\alpha(x) & =k g^{(1)}(x)=k\left[\psi_{1}(x)-\theta\right], \\
\alpha^{\prime}(x)= & \delta_{k}\left[(k-1) g_{(k-1)}^{(1)}(x)-k g^{(1)}(x)\right] \\
= & \delta_{k}\left[\psi_{2}(x, x)+(k-2) E\left[\psi_{3}\left(x, X_{2}, X_{2}\right)\right]-(k-1) \theta_{k-1}-k g^{(1)}(x)\right], \\
\beta(x, y)= & k(k-1) g^{(2)}(x, y)=k(k-1)\left[\psi_{2}(x, y)-\psi_{1}(x)-\psi_{1}(y)+\theta\right], \\
\gamma(x, y, z)= & k(k-1)(k-2) g^{(3)}(x, y, z) \\
= & k(k-1)(k-2)\left[\psi_{3}(x, y, z)-\psi_{2}(x, y)-\psi_{2}(x, z)-\psi_{2}(y, z)\right. \\
& \left.+\psi_{1}(x)+\psi_{1}(y)+\psi_{1}(z)-\theta\right] .
\end{aligned}
$$

Then we can write $Y_{n}^{* *}$ as

$$
\begin{aligned}
Y_{n}^{* *}= & \sum_{i=1}^{n}\left[\frac{\alpha\left(X_{i}\right)}{n^{1 / 2}}+\frac{\alpha^{\prime}\left(X_{i}\right)}{n^{3 / 2}}\right]+\sum_{1 \leq i<j \leq n} \frac{\beta\left(X_{i}, X_{j}\right)}{n^{3 / 2}} \\
& +\sum_{1 \leq i<j<l \leq n} \frac{\gamma\left(X_{i}, X_{j}, X_{l}\right)}{n^{5 / 2}} .
\end{aligned}
$$

The Edgeworth expansion of the statistic given by this form has been derived by Lai and Wang (1993). We will derive the Edgeworth expansion of $Y_{n}$ by using their result. Their conditions (A2) and (A3) are satisfied by the properties of H-decomposition. The condition (A1) is not related to our situation. Conditions (C) or (D) are described as follows by our notations for the one-dimensional random variable:

Condition (C). $E\left|g^{(2)}\right|^{r}<\infty$ for some $r>2$ and there exists $K$ Borel functions $f_{j}: R \rightarrow R$ such that $K(r-2)>8(4 r-5), E f_{j}^{2}\left(X_{1}\right)<\infty(j=$ $1, \ldots, K)$, and the covariance matrix of $\left(W_{1}, \ldots, W_{K}\right)$ is positive definite, where $W_{j}=\left(L f_{j}\right)\left(X_{1}\right)$ and $(L f)(y)=E\left[g^{(2)}\left(y, X_{2}\right) f\left(X_{2}\right)\right]$.

The original condition contains the term $I_{\left[E\left|g^{(3)}\left(X_{1}, X_{2}, X_{3}\right)\right|>0\right]}$, which equals 1 since $g^{(3)}\left(X_{1}, X_{2}, X_{3}\right)$ is not likely to be zero under the assumption that the kernel $g$ is not degenerate, that is, $\sigma_{1}^{2}>0$. It also contains $E\left|g^{(2)}\right|^{r}<\infty$ for some $r>2$. This condition is satisfied with $r=4$ under our condition $E\left[\left|\psi_{3}\left(X_{1}, X_{2}, X_{3}\right)\right|^{4}\right]<$ $\infty$ of Theorem 3.2, which is assumed for the condition (A4) of Lai and Wang (1993).

Condition (D). There exist constants $c_{j}$ and Borel functions $f_{j}: R \rightarrow R$ such that $E f_{j}(X)=0, E\left|f_{j}(X)\right|^{r}<\infty$ for some $r \geq 5$ and $g^{(2)}=$ $\sum_{j=1}^{K} c_{j} f_{j}\left(X_{1}\right) f_{j}\left(X_{2}\right)$ a.s.; moreover, for some $0<\varepsilon<\min \left\{1,2\left(1-11 r^{-1} / 3\right)\right\}$,

$$
\limsup _{|t| \rightarrow \infty} \sup _{\left|s_{1}\right|+\cdots+\left|s_{K}\right| \leq|t|^{-\varepsilon}}\left|E \exp \left(i t\left\{\alpha(X)+\sum_{j=1}^{K} s_{j} f_{j}(X)\right\}\right)\right|<1 .
$$


We shall use the same notations as Lai and Wang (1993) for constants $\sigma, a_{3}$, $a_{4}, a^{\prime}, b, c, \kappa_{3}, \kappa_{4}$. In our situation these constants are given by as follows:

$$
\begin{aligned}
& \sigma^{2}=k^{2} \sigma_{1}^{2}, \quad\left(\sigma_{1}^{2}=\operatorname{Var}\left[\psi_{1}(X)\right]\right), \\
& a_{3}=k^{3} E\left[\left(g^{(1)}(X)\right)^{3}\right]=k^{3} E\left[\left(\psi_{1}(X)-\theta\right)^{3}\right] \text {, } \\
& a_{4}=k^{4} E\left[\left(g^{(1)}(X)\right)^{4}\right]=k^{4} E\left[\left(\psi_{1}(X)-\theta\right)^{4}\right] \text {, } \\
& a^{\prime}=k \delta_{k}\left\{(k-1) E\left[g^{(1)}(X) g_{(k-1)}^{(1)}(X)\right]-k E\left[g^{(1)}(X)\right]^{2}\right\} \\
& =k \delta_{k}\left\{(k-1) E\left[g^{(1)}(X) g_{(k-1)}^{(1)}(X)\right]-k \sigma_{1}^{2}\right\} \\
& =k \delta_{k}\left\{E\left[\psi_{1}\left(X_{1}\right) \psi_{2}\left(X_{1}, X_{1}\right)\right]+(k-2) E\left[\psi_{1}\left(X_{1}\right) \psi_{3}\left(X_{1}, X_{2}, X_{2}\right)\right]\right. \\
& \left.-(k-1) \theta_{k-1} \theta-k \sigma_{1}^{2}\right\} \text {, } \\
& b=k^{3}(k-1) E\left\{g^{(1)}\left(X_{1}\right) g^{(1)}\left(X_{2}\right) g^{(2)}\left(X_{1}, X_{2}\right)\right\} \\
& =k^{3}(k-1)\left\{E\left[\psi_{1}\left(X_{1}\right) \psi_{1}\left(X_{2}\right) \psi_{2}\left(X_{1}, X_{2}\right)\right]-2 \sigma_{1}^{2} \theta-\theta^{3}\right\}, \\
& c=k^{4}(k-1)(k-2) E\left\{g^{(1)}\left(X_{1}\right) g^{(1)}\left(X_{2}\right) g^{(1)}\left(X_{3}\right) g^{(3)}\left(X_{1}, X_{2}, X_{3}\right)\right\} \\
& =k^{4}(k-1)(k-2)\left\{E\left[\psi_{1}\left(X_{1}\right) \psi_{1}\left(X_{2}\right) \psi_{1}\left(X_{3}\right) \psi_{3}\left(X_{1}, X_{2}, X_{3}\right)\right]\right. \\
& \left.-3 \theta E\left[\psi_{1}\left(X_{1}\right) \psi_{1}\left(X_{2}\right) \psi_{2}\left(X_{1}, X_{2}\right)\right]+3 \sigma_{1}^{2} \theta^{2}+2 \theta^{4}\right\} \text {, } \\
& \kappa_{3}=a_{3}+3 b \\
& =k^{3}\left\{E\left[\left(g^{(1)}(X)\right)^{3}\right]+3(k-1) E\left[g^{(1)}\left(X_{1}\right) g^{(1)}\left(X_{2}\right) g^{(2)}\left(X_{1}, X_{2}\right)\right]\right\}, \\
& =k^{3}\left\{E\left[\left(\psi_{1}(X)\right)^{3}\right]+E\left[\psi_{1}\left(X_{1}\right) \psi_{1}\left(X_{2}\right) \psi_{2}\left(X_{1}, X_{2}\right)\right]\right. \\
& \left.-3(2 k-1) \sigma_{1}^{2} \theta-(3 k-2) \theta^{3}\right\} \text {, } \\
& \kappa_{4}=a_{4}-3 \sigma^{4}+4 c+12 k^{4}(k-1) E\left[\left[g^{(1)}\left(X_{1}\right)\right]^{2} g^{(1)}\left(X_{2}\right) g^{(2)}\left(X_{1}, X_{2}\right)\right] \\
& +12 k^{4}(k-1)^{2} E\left[g^{(1)}\left(X_{1}\right) g^{(1)}\left(X_{2}\right) g^{(2)}\left(X_{1}, X_{3}\right) g^{(2)}\left(X_{2}, X_{3}\right)\right] \\
& =a_{4}-3 \sigma^{4}+4 c+12 k^{4}(k-1)\left\{E\left[\left[\psi_{1}\left(X_{1}\right)\right]^{2} \psi_{1}\left(X_{2}\right) \psi_{2}\left(X_{1}, X_{2}\right)\right]\right. \\
& +(k-1) E\left[\psi_{1}\left(X_{1}\right) \psi_{1}\left(X_{2}\right) \psi_{2}\left(X_{1}, X_{3}\right) \psi_{2}\left(X_{2}, X_{3}\right)\right] \\
& -2 k \theta E\left[\psi_{1}\left(X_{1}\right) \psi_{1}\left(X_{2}\right) \psi_{2}\left(X_{1}, X_{2}\right)\right]-\theta E\left(\left[\psi_{1}(X)-\theta\right]^{3}\right) \\
& \left.+k \theta^{4}+(k-1) \theta^{2} \sigma_{1}^{2}-k \sigma_{1}^{4}\right\} \text {. }
\end{aligned}
$$

The constants $\sigma, \sigma_{1}, a^{\prime}, \kappa_{3}, \kappa_{4}$ among the aboves, which are necessary for the Edgeworth expansion of Y-statistic, are calculated by using $\delta_{k}$ and the following quantities based on the kernel $g$ :

$$
\begin{aligned}
& \theta\left(=E\left[g\left(X_{1}, \ldots, X_{k}\right)\right]\right), \quad \theta_{k-1}\left(=E\left[g\left(X_{1}, X_{1}, X_{2}, \ldots, X_{k-1}\right)\right]\right), \quad E\left[\psi_{1}^{2}(X)\right], \\
& E\left[\psi_{1}^{3}(X)\right], \quad E\left[\psi_{1}^{4}(X)\right], \quad E\left[\psi_{1}\left(X_{1}\right) \psi_{2}\left(X_{1}, X_{1}\right)\right], \quad E\left[\psi_{1}\left(X_{1}\right) \psi_{3}\left(X_{1}, X_{2}, X_{2}\right)\right], \\
& E\left[\psi_{1}\left(X_{1}\right) \psi_{1}\left(X_{2}\right) \psi_{2}\left(X_{1}, X_{2}\right)\right], \quad E\left[\psi_{1}\left(X_{1}\right) \psi_{1}\left(X_{2}\right) \psi_{1}\left(X_{3}\right) \psi_{3}\left(X_{1}, X_{2}, X_{3}\right)\right], \\
& \left.E\left\{\left[\psi_{1}\left(X_{1}\right)\right]^{2} \psi_{1}\left(X_{2}\right) \psi_{2}\left(X_{1}, X_{2}\right)\right]\right\}, \quad E\left[\psi_{1}\left(X_{1}\right) \psi_{1}\left(X_{2}\right) \psi_{2}\left(X_{1}, X_{3}\right) \psi_{2}\left(X_{2}, X_{3}\right)\right] .
\end{aligned}
$$

Let $\phi$ and $\Phi$ be the density function and the distribution function of the standard normal distribution $N(0,1)$, respectively. We put the functions $P_{1}, P_{2}, P_{21}, P_{22}$ and $G_{n}$ as follows:

$$
P_{1}(z)=\frac{\kappa_{3}}{6 \sigma^{3}}\left(z^{2}-1\right)=\frac{\kappa_{3}}{6 k^{3} \sigma_{1}^{3}}\left(z^{2}-1\right),
$$




$$
\begin{aligned}
& P_{2}(z)=P_{21}(z)+P_{22}(z), \\
& P_{21}(z)=\left[a^{\prime}+\frac{1}{4} k^{2}(k-1)^{2}\left[\sigma_{2}^{2}-2 \sigma_{1}^{2}\right]\right] \frac{z}{\sigma^{2}}, \\
& P_{22}(z)=\frac{\kappa_{4}}{24 \sigma^{4}}\left(z^{3}-3 z\right)+\frac{\kappa_{3}^{2}}{72 \sigma^{6}}\left(z^{5}-10 z^{3}+15 z\right), \\
& G_{n}(z)=\Phi(z)-\frac{1}{n^{1 / 2}} \phi(z) P_{1}(z)-\frac{1}{n} \phi(z) P_{2}(z),
\end{aligned}
$$

where $\sigma_{2}^{2}=\operatorname{Var}\left[\psi_{2}\left(X_{1}, X_{2}\right)\right]>0$. Under certain conditions given by Lai and Wang (1993), we have Edgeworth expansion of $Y_{n}^{* *}$ such that

$$
\sup _{-\infty<z<\infty}\left|P\left(\frac{Y_{n}^{* *}}{\sigma} \leq z\right)-G_{n}(z)\right|=o\left(\frac{1}{n}\right) .
$$

Therefore we have

$$
\sup _{-\infty<z<\infty}\left|P\left(\frac{1}{\sigma}\left(Y_{n}^{* *}+\frac{\mu_{k}}{\sqrt{n}}\right) \leq z\right)-G_{n}\left(z-\frac{\mu_{k}}{\sigma \sqrt{n}}\right)\right|=o\left(\frac{1}{n}\right) .
$$

Now we shall compute

$$
\begin{aligned}
G_{n}\left(z-\frac{\mu_{k}}{\sigma \sqrt{n}}\right)= & \Phi\left(z-\frac{\mu_{k}}{\sigma \sqrt{n}}\right)-\frac{1}{\sqrt{n}} \phi\left(z-\frac{\mu_{k}}{\sigma \sqrt{n}}\right) P_{1}\left(z-\frac{\mu_{k}}{\sigma \sqrt{n}}\right) \\
& -\frac{1}{n} \phi\left(z-\frac{\mu_{k}}{\sigma \sqrt{n}}\right) P_{2}\left(z-\frac{\mu_{k}}{\sigma \sqrt{n}}\right) .
\end{aligned}
$$

LEMMA 3.1.

$$
\sup _{-\infty<z<\infty}\left|G_{n}\left(z-\frac{\mu_{k}}{\sigma \sqrt{n}}\right)-G_{n}^{*}(z)\right|=O\left(n^{-3 / 2}\right),
$$

where

$$
\begin{aligned}
& G_{n}^{*}(z)=\Phi(z)-\frac{1}{\sqrt{n}} \phi(z) P_{1}^{*}(z)-\frac{1}{n} \phi(z) P_{2}^{*}(z) \\
& P_{1}^{*}(z)=\frac{\mu_{k}}{\sigma}+P_{1}(z)=\frac{\mu_{k}}{\sigma}+\frac{\kappa_{3}}{6 \sigma^{3}}\left(z^{2}-1\right)
\end{aligned}
$$

and

$$
\begin{aligned}
P_{2}^{*}(z) & =P_{21}^{*}(z)+P_{22}(z) \\
P_{21}^{*}(z) & =\frac{\mu_{k}^{2}}{2 \sigma^{2}} z+\frac{\mu_{k} \kappa_{3}}{6 \sigma^{4}}\left(z^{3}-3 z\right)+P_{21}(z) \\
& =\left[\frac{\mu_{k}^{2}}{2}+a^{\prime}+\frac{1}{4} k^{2}(k-1)^{2}\left[\sigma_{2}^{2}-2 \sigma_{1}^{2}\right]\right] \frac{1}{\sigma^{2}} z+\frac{\mu_{k} \kappa_{3}}{6 \sigma^{4}}\left(z^{3}-3 z\right) .
\end{aligned}
$$

That is,

$$
\begin{aligned}
P_{2}^{*}(z)= & {\left[\frac{\mu_{k}^{2}}{2}+a^{\prime}+\frac{1}{4} k^{2}(k-1)^{2}\left[\sigma_{2}^{2}-2 \sigma_{1}^{2}\right]\right] \frac{1}{\sigma^{2}} z } \\
& +\left[\frac{\mu_{k} \kappa_{3}}{6}+\frac{\kappa_{4}}{24}\right] \frac{1}{\sigma^{4}}\left(z^{3}-3 z\right)+\frac{\kappa_{3}^{2}}{72 \sigma^{6}}\left(z^{5}-10 z^{3}+15 z\right) .
\end{aligned}
$$


Proof. By applying the Taylor expansion and noting $\sup _{z}|z|^{j} \phi(z)<\infty$ $(j=1,2, \ldots)$, we have

$$
\sup _{z}\left|\Phi\left(z-\frac{\mu_{k}}{\sigma \sqrt{n}}\right)-\left\{\Phi(z)-\frac{\mu_{k}}{\sigma \sqrt{n}} \phi(z)-\frac{\mu_{k}^{2}}{2 \sigma^{2} n} z \phi(z)\right\}\right|=O\left(n^{-3 / 2}\right)
$$

and

$$
\sup _{z}\left|\phi\left(z-\frac{\mu_{k}}{\sigma \sqrt{n}}\right)-\left\{\phi(z)+\frac{\mu_{k}}{\sigma \sqrt{n}} z \phi(z)+\frac{\mu_{k}^{2}}{2 \sigma^{2} n}\left(z^{2}-1\right) \phi(z)\right\}\right|=O\left(n^{-3 / 2}\right) .
$$

Therefore by the finiteness of $\sup _{z}|z|^{j} \phi(z)(j=1,2, \ldots)$, we have

and

$$
\begin{aligned}
\sup _{z} \mid \phi\left(z-\frac{\mu_{k}}{\sigma \sqrt{n}}\right) P_{1}\left(z-\frac{\mu_{k}}{\sigma \sqrt{n}}\right) \\
\quad-\frac{\kappa_{3}}{6 \sigma^{3}}\left\{\left(z^{2}-1\right) \phi(z)+\frac{\mu_{k}}{\sigma \sqrt{n}}\left(z^{3}-3 z\right) \phi(z)\right\} \mid=O\left(n^{-1}\right)
\end{aligned}
$$

$$
\sup _{z}\left|\phi\left(z-\frac{\mu_{k}}{\sigma \sqrt{n}}\right) P_{2}\left(z-\frac{\mu_{k}}{\sigma \sqrt{n}}\right)-\phi(z) P_{2}(z)\right|=O\left(n^{-1 / 2}\right) .
$$

Hence applying (3.6), (3.7) and (3.8) to the right-hand side of (3.3), we get $(3.4)$.

Thus by (2.12), (3.2) and (3.4) we get the following.

TheOrem 3.2. We assume that $d(k, k)>0, \sigma_{1}^{2}>0$,

$$
\begin{aligned}
& E\left[\left|g\left(X_{j_{1}}, \ldots, X_{j_{k}}\right)\right|^{2}\right]<\infty \text { for } 1 \leq j_{1} \leq \cdots \leq j_{k} \leq k \\
& E\left|\psi_{1}\left(X_{1}\right)\right|^{4}<\infty, \quad E\left|\psi_{2}\left(X_{1}, X_{2}\right)\right|^{4}<\infty, \quad E\left|\psi_{2}\left(X_{1}, X_{1}\right)\right|^{3}<\infty
\end{aligned}
$$

and

$$
E\left|\psi_{3}\left(X_{1}, X_{2}, X_{2}\right)\right|^{2}<\infty, \quad E\left|\psi_{3}\left(X_{1}, X_{2}, X_{3}\right)\right|^{4}<\infty .
$$

Suppose that

$$
\limsup _{|t| \rightarrow \infty}\left|E e^{i t \psi_{1}\left(X_{1}\right)}\right|<1
$$

and either Condition $(C)$ or $(D)$ holds. Then we have

$$
\sup _{-\infty<z<\infty}\left|P\left(\frac{\sqrt{n}}{k \sigma_{1}}\left(Y_{n}-\theta\right) \leq z\right)-G_{n}^{*}(z)\right|=o\left(n^{-1}\right)
$$

where $G_{n}^{*}(z)$ is given by (3.5).

Let us put

$$
G_{U, n}^{*}(z)=\Phi(z)-\left[\frac{1}{\sqrt{n}} \phi(z) P_{1}(z)+\frac{1}{n} \phi(z) P_{22}(z)\right]
$$


which is the Edgeworth expansion of $\sqrt{n}\left(U_{n}-\theta\right) /\left(k \sigma_{1}\right)$ with remainder $o\left(n^{-1}\right)$ (see Maesono (1987)). Then by (3.5) we can write

$$
G_{n}^{*}(z)=G_{U, n}^{*}(z)-\left[\frac{\mu_{k}}{\sqrt{n} \sigma} \phi(z)+\frac{1}{n} \phi(z) P_{21}^{*}(z)\right] .
$$

The second term of the right-hand side of this equation shows the difference between the Edgeworth expansions of the Y-statistic and U-statistic with remainder $o\left(n^{-1}\right)$. As seen in (3.11), the effect of the weight function $w$ appears only in terms of $\mu_{k}$ and $a^{\prime}$, where $P_{21}^{*}$ includes $\mu_{k}$ and $a^{\prime}$. For the V-statistic $V_{n}$ and S-statistic $S_{n}, \delta_{k}=k(k-1) / 2$. Hence, for $V_{n}$ and $S_{n}$,

$$
\mu_{k}=\frac{k(k-1)}{2}\left(\theta_{k-1}-\theta\right), \quad a^{\prime}=\frac{k^{2}(k-1)}{2}\left[(k-1) E\left[g^{(1)}(X) g_{(k-1)}^{(1)}(X)\right]-k \sigma_{1}^{2}\right] \text {. }
$$

The Edgeworth expansion of $V_{n}$ is also derived by Takahashi (1988) using a different method. For the LB-statistic $B_{n}, \delta_{k}=k(k-1)$, and

$$
\mu_{k}=k(k-1)\left(\theta_{k-1}-\theta\right), \quad a^{\prime}=k^{2}(k-1)\left[(k-1) E\left[g^{(1)}(X) g_{(k-1)}^{(1)}(X)\right]-k \sigma_{1}^{2}\right] .
$$

Using a similar method to the above, we can easily derive the second-order expansion. Taking the similar method to Proposition 2.1, we have the following. We suppose (2.4). Then we have

$$
\sqrt{n}\left(Y_{n}-\theta\right)=\sqrt{n}\left(U_{n}-\theta\right)+\frac{\mu_{k}}{\sqrt{n}}+R_{n}^{\prime \prime}
$$

where $E\left|R_{n}^{\prime \prime}\right|^{2}=O\left(n^{-3 / 2}\right)$. By (2.10), we have

$$
\begin{aligned}
& \sup _{-\infty<z<\infty}\left|P\left(\frac{\sqrt{n}}{\sigma}\left(Y_{n}-\theta\right) \leq z\right)-P\left(\frac{\sqrt{n}}{\sigma}\left(U_{n}-\theta\right)+\frac{\mu_{k}}{\sigma \sqrt{n}} \leq z\right)\right| \\
& =O\left(n^{-3 / 4}\right) .
\end{aligned}
$$

Under certain conditions which are given in the following Proposition 3.3, we have

$$
\sup _{-\infty<z<\infty}\left|P\left(\frac{\sqrt{n}}{\sigma}\left(U_{n}-\theta\right) \leq z\right)-G_{U, n}^{\prime}(z)\right|=o\left(n^{-1 / 2}\right)
$$

where

$$
G_{U, n}^{\prime}(z)=\Phi(z)-\frac{1}{\sqrt{n}} \phi(z) P_{1}(z)
$$

(see Maesono and Yamato (1994), Theorem 2.2). Therefore we have

$$
\sup _{z}\left|P\left(\frac{\sqrt{n}}{\sigma}\left(U_{n}-\theta\right)+\frac{\mu_{k}}{\sigma \sqrt{n}} \leq z\right)-G_{U, n}^{\prime}\left(z-\frac{\mu_{k}}{\sigma \sqrt{n}}\right)\right|=o\left(n^{-1 / 2}\right) .
$$


Applying (3.6) and (3.7) to $G_{U, n}^{\prime}\left(z-\mu_{k} /(\sigma \sqrt{n})\right)$,

$$
\begin{aligned}
& \sup _{z}\left|G_{U, n}^{\prime}\left(z-\frac{\mu_{k}}{\sigma \sqrt{n}}\right)-\left[\Phi(z)-\frac{1}{\sqrt{n}}\left[\frac{\mu_{k}}{\sigma}+\frac{\kappa_{3}}{6 \sigma^{3}}\left(z^{2}-1\right)\right] \phi(z)\right]\right| \\
& =O\left(n^{-1}\right) .
\end{aligned}
$$

From (3.13), (3.14), (3.15) and (3.16), we have the second-order Edgeworth expansion.

Proposition 3.3. We assume that $d(k, k)>0, \sigma_{1}>0, E \mid g\left(X_{1}, \ldots\right.$, $\left.X_{k}\right)\left.\right|^{3}<\infty$, and $E\left[\left|g\left(X_{j_{1}}, \ldots, X_{j_{k}}\right)\right|^{2}\right]<\infty$ for $1 \leq j_{1} \leq \cdots \leq j_{k} \leq k$. Suppose that $\lim \sup _{|t| \rightarrow \infty}\left|E e^{i t \psi_{1}\left(X_{1}\right)}\right|<1$. Then

$$
\sup _{-\infty<z<\infty}\left|P\left(\frac{\sqrt{n}}{k \sigma_{1}}\left(Y_{n}-\theta\right) \leq z\right)-G_{n}^{\prime \prime}(z)\right|=o\left(n^{-1 / 2}\right)
$$

where

$$
G_{n}^{\prime \prime}(z)=\Phi(z)-\frac{1}{\sqrt{n}}\left[\frac{\mu_{k}}{\sigma}+\frac{\kappa_{3}}{6 \sigma^{3}}\left(z^{2}-1\right)\right] \phi(z)
$$

We can write

$$
G_{n}^{\prime \prime}(z)=G_{U, n}^{\prime}(z)-\frac{\mu_{k}}{\sqrt{n} \sigma} \phi(z) .
$$

That is, the difference between the Edgeworth expansions of the standardized Y-statistic and U-statistic is $\left[\mu_{k} /(\sqrt{n} \sigma)\right] \phi(z)$, with remainder $o\left(n^{-1 / 2}\right)$.

\section{Studentized Y-statistic}

Using the results of Maesono $(1995,1996)$ we shall evaluate the Edgeworth expansion of the studentized Y-statistic with a remainder term $o\left(n^{-1 / 2}\right)$. For studentization of the Y-statistic, we consider the jackknife variance estimator $\hat{\sigma}_{n}^{2}$ of $\sqrt{n} Y_{n}$ given by

$$
\hat{\sigma}_{n}^{2}=(n-1) \sum_{i=1}^{n}\left(Y_{n}^{(i)}-Y_{n}\right)^{2},
$$

where for $i=1, \ldots, n, Y_{n}^{(i)}$ is the Y-statistic computed from a sample of size $n-1$ with $X_{i}$ left out. We put

$$
f^{*}(x)=\frac{1}{2}\left(\left[g^{(1)}(x)\right]^{2}-\sigma_{1}^{2}\right)+(k-1) E\left[g^{(1)}\left(X_{2}\right) g^{(2)}\left(x, X_{2}\right)\right] .
$$

Thus by Maesono (1996, p. 205) we have the asymptotic evaluation of $\hat{\sigma}_{n}^{-1}$ as follows: 
Lemma 4.1. We suppose that $E\left|g\left(X_{1}, \ldots, X_{k}\right)\right|^{4+\varepsilon}<\infty$ and $E\left|g_{(k-1)}\left(X_{1}, \ldots, X_{k-1}\right)\right|^{4+\varepsilon}<\infty$, for $\varepsilon>0, \sigma_{1}^{2}>0$, and $E\left|g_{(j)}\left(X_{1}, \ldots, X_{j}\right)\right|^{2}<$ $\infty$ for $j=1,2, \ldots, k-2$. Then we have

$$
k \sigma_{1} \hat{\sigma}_{n}^{-1}=\sigma \hat{\sigma}_{n}^{-1}=1-\frac{1}{n \sigma_{1}^{2}} \sum_{i=1}^{n} f^{*}\left(X_{i}\right)+R_{1, n},
$$

where $E\left|R_{1, n}\right|^{2}=O\left(n^{-2}\right)$.

We note that relation (4.3) also holds for the jackknife estimator based on the U-statistic $U_{n}$ with the same order with respect to the error term; from Lemma 4 of Maesono (1995). Then, by Theorem 3 of Maesono (1995), we have the following.

Lemma 4.2. We suppose that $E\left|g\left(X_{1}, \ldots, X_{k}\right)\right|^{4+\varepsilon}<\infty$ for $\varepsilon>0, \sigma_{1}^{2}>$ 0 , and $\lim \sup _{|t| \rightarrow \infty}\left|E\left[\exp \left\{i t g^{(1)}(X)\right\}\right]\right|<1$. Then

$$
\sup _{-\infty<z<\infty}\left|P\left(\sqrt{n} \hat{\sigma}_{n}^{-1}\left(U_{n}-\theta\right) \leq z\right)-H_{n}(z)\right|=o\left(n^{-1 / 2}\right),
$$

where

$$
H_{n}(z)=\Phi(z)+\frac{1}{\sqrt{n}} \frac{1}{6 \sigma^{3}}\left[a_{3} z^{2}+\kappa_{3}\left(z^{2}+1\right)\right] \phi(z) .
$$

From (4.3) we have

$$
\frac{1}{\sqrt{n}} \mu_{k} \hat{\sigma}_{n}^{-1}=\frac{\mu_{k}}{\sigma \sqrt{n}}+R_{2, n}
$$

where $E\left|R_{2, n}\right|^{2}=O\left(n^{-2}\right)$. Therefore by (3.12) and (4.5) we have

$$
\sqrt{n} \hat{\sigma}_{n}^{-1}\left(Y_{n}-\theta\right)=\sqrt{n} \hat{\sigma}_{n}^{-1}\left(U_{n}-\theta\right)+\frac{\mu_{k}}{\sigma \sqrt{n}}+R_{3, n},
$$

where $E\left|R_{3, n}\right|^{2}=O\left(n^{-3 / 2}\right)$. We note that

$$
H_{n}\left(z-\frac{\mu_{k}}{\sigma \sqrt{n}}\right)=H_{n}^{*}(z)+O\left(\frac{1}{n}\right),
$$

where

$$
\begin{aligned}
H_{n}^{*}(z) & =H_{n}(z)-\frac{\mu_{k}}{\sqrt{n} \sigma} \phi(z) \\
& =\Phi(z)+\frac{1}{\sqrt{n}}\left(-\frac{\mu_{k}}{\sigma}+\frac{1}{6 \sigma^{3}}\left[a_{3} z^{2}+\kappa_{3}\left(z^{2}+1\right)\right]\right) \phi(z) .
\end{aligned}
$$

Thus by the similar method to the last section, from (4.4), (4.6) and (4.7) we have the following. 
Proposition 4.3. We suppose that $E\left|g\left(X_{1}, \ldots, X_{k}\right)\right|^{4+\varepsilon}<\infty$ and $E\left|g_{(k-1)}\left(X_{1}, \ldots, X_{k-1}\right)\right|^{4+\varepsilon}<\infty$, for $\varepsilon>0$, and $E\left|g_{(j)}\left(X_{1}, \ldots, X_{j}\right)\right|^{2}<\infty$ for $j=1,2, \ldots, k-2$. We also suppose that $\sigma_{1}^{2}>0$, and

$$
\limsup _{|t| \rightarrow \infty}\left|E\left[\exp \left\{i g^{(1)}(X)\right\}\right]\right|<1 \text {. }
$$

Then

$$
\sup _{-\infty<z<\infty}\left|P\left(\sqrt{n} \hat{\sigma}_{n}^{-1}\left(Y_{n}-\theta\right) \leq z\right)-H_{n}^{*}(z)\right|=o\left(n^{-1 / 2}\right)
$$

As seen in (4.7), the difference between the Edgeworth expansions of the studentized Y-statistic and U-statistic using a jackknife variance estimator is $\left[\mu_{k} /(\sqrt{n} \sigma)\right] \phi(z)$ with remainder $o\left(n^{-1 / 2}\right)$. With the remainder $o\left(n^{-1 / 2}\right)$, this difference is as same as the difference between the Edgeworth expansions of the standardized Y-statistic and U-statistic which is given by (3.18).

We used the the jackknife variance estimator for studentization of Y-statistic and obtained its Edgeworth expansion with a remainder of term $o\left(n^{-1 / 2}\right)$. We shall discuss the Edgeworth expansion by using other estimators and/or with a remainder of term $o\left(n^{-1}\right)$ in a future paper.

\section{Examples}

From (3.11), the difference between the Edgeworth expansions of standardized Y-statistic and U-statistic with remainder $o\left(n^{-1}\right)$ can be written as

$$
\begin{aligned}
& G_{n}^{*}(z)-G_{U, n}^{*}(z) \\
& =-\left\{\frac{1}{\sqrt{n} \sigma} A_{1} \phi(z)+\frac{1}{n \sigma^{2}} A_{2} z \phi(z)+\frac{1}{6 n \sigma^{4}} A_{3}\left(z^{3}-3 z\right) \phi(z)\right\},
\end{aligned}
$$

where

$$
A_{1}=\mu_{k}, \quad A_{2}=\frac{\mu_{k}^{2}}{2}+a^{\prime}+\frac{1}{4} k^{2}(k-1)^{2}\left[\sigma_{2}^{2}-2 \sigma_{1}^{2}\right], \quad A_{3}=\mu_{k} \kappa_{3} .
$$

We show two examples of the expansions $G_{n}^{*}(z)$, by giving $G_{U, n}^{*}(z)$ and $A_{1}, A_{2}$, $A_{3}$. Incidentally, we also give the expansions $G_{n}^{\prime \prime}, G_{U, n}^{*}, H_{n}$ and $H_{n}^{*}$.

(1) At first we consider the third central moment $\theta=\int(x-\mu)^{3} d F(x)$, where $\mu$ is the mean of $F$. Its kernel $g\left(x_{1}, x_{2}, x_{3}\right)$ is given by

$$
\frac{1}{3}\left(x_{1}^{3}+x_{2}^{3}+x_{3}^{3}\right)-\frac{1}{2}\left(x_{1}^{2} x_{2}+x_{1}^{2} x_{3}+x_{1} x_{2}^{2}+x_{2}^{2} x_{3}+x_{1} x_{3}^{2}+x_{2} x_{3}^{2}\right)+2 x_{1} x_{2} x_{3} .
$$

We assume that the distribution $F$ has a density, it is symmetric about zero and its mean is zero. We also assume $E|X|^{12+\varepsilon}<\infty(0<\varepsilon<1)$ for $X$ having the distribution $F$ and denote its moments about the origin by $m^{\prime}{ }_{j}(j=2,4,6,8,10,12)$. We can write $g^{(2)}\left(X_{1}, X_{2}\right)=\left(x_{1}+x_{2}\right)\left(m_{2}^{\prime}-x_{1} x_{2}\right) / 2=\sum_{j=1}^{5} c_{j} f_{j}\left(X_{1}\right) f_{j}\left(X_{2}\right)$, where

$$
c_{1}=\frac{1}{2}, \quad c_{2}=-\frac{1}{2}, \quad c_{3}=\frac{1}{2}\left(1-m_{2}^{\prime}\right), \quad c_{4}=\frac{1}{2} m_{2}^{\prime}, \quad c_{5}=-\frac{1}{2} m_{2}^{\prime},
$$


and

$$
f_{1}(x)=x^{2}, \quad f_{2}(x)=x^{2}+x, \quad f_{3}(x)=x, \quad f_{4}(x)=x+1, \quad f_{5}(x)=1 .
$$

We note that

$$
\left|E e^{i t\left[\alpha(X)+\Sigma_{j=1}^{5} s_{j} f_{j}(X)\right]}\right| \leq|I|+\left|E e^{i t \alpha(X)}\right|,
$$

where

$$
I=E e^{i t \alpha(X)}\left\{e^{i t \Sigma_{j=1}^{5} s_{j} f_{j}(X)}-1\right\} .
$$

Then, it follows that under the condition $\left|s_{1}\right|+\cdots+\left|s_{5}\right| \leq|t|^{-\delta}(0<\forall \delta<1)$,

$$
|I| \leq E\left|e^{i t \Sigma_{j=1}^{5} s_{j} f_{j}(X)}-1\right| \leq|t|^{-\delta}\left(E X^{2}+E|X|+1\right),
$$

which tends to zero as $|t| \rightarrow \infty$. Since $X$ has the density, $E e^{i t \alpha(X)}=E e^{i t\left(X^{3}-3 X\right)} \rightarrow$ 0 as $t \rightarrow \infty$. Thus the condition (D) is satisfied.

Now, we have

$$
\begin{aligned}
& \sigma_{1}^{2}=\frac{1}{9}\left(m^{\prime}{ }_{6}-6 m^{\prime}{ }_{4} m^{\prime}{ }_{2}+9 m_{2}^{\prime 3}\right), \\
& \sigma_{2}^{2}=\frac{2}{9} m^{\prime}{ }_{6}-\frac{5}{6} m^{\prime}{ }_{4} m_{2}^{\prime}+\frac{3}{2} m_{2}^{\prime 3}, \\
& \sigma^{2}=m_{6}^{\prime}-6 m^{\prime}{ }_{4} m_{2}^{\prime}+9 m_{2}^{\prime 3} \text {, } \\
& a_{3}=0 \text {, } \\
& a_{4}=m^{\prime}{ }_{12}-12 m^{\prime}{ }_{10} m^{\prime}{ }_{2}+54 m^{\prime}{ }_{8} m_{2}^{\prime 2}-108 m^{\prime}{ }_{6} m_{2}^{\prime 3}+81 m^{\prime}{ }_{4} m_{2}^{\prime 4} \text {, } \\
& a^{\prime}=-\delta_{3}\left(m_{6}^{\prime}-6 m^{\prime}{ }_{4} m_{2}+9 m_{2}^{\prime 3}\right) \text {, } \\
& b=0 \text {, } \\
& c=12\left(m^{\prime}{ }_{4}-3 m_{2}^{\prime 2}\right)^{3}, \\
& \kappa_{3}=0 \text {, } \\
& \kappa_{4}=m^{\prime}{ }_{12}-12 m^{\prime}{ }_{10} m^{\prime}{ }_{2}-36 m^{\prime}{ }_{8} m_{4}^{\prime}+162 m^{\prime}{ }_{8} m_{2}^{\prime 2}-3 m_{6}^{\prime 2}+360 m^{\prime}{ }_{6} m_{4}^{\prime} m_{2}^{\prime} \\
& -1134 m_{6}^{\prime} m_{2}^{\prime 3}+156 m_{4}^{\prime 3}-2268 m^{\prime 2}{ }_{4}^{\prime 2}{ }_{2}^{2}+5913 m_{4}^{\prime} m_{2}^{\prime 4}-3483 m_{2}^{\prime 6} \text {, } \\
& \mu_{k}=0 \text {. }
\end{aligned}
$$

The coefficients necessary for the Edgeworth expansion are calculated by using $\delta_{3}$ and the moments $m_{2}^{\prime}, m_{4}^{\prime}, m_{6}^{\prime}, m_{8}^{\prime}, m_{10}^{\prime}, m_{12}^{\prime}$. In this case, we have $P_{1}(z)=0$, $P_{22}(z)=\kappa_{4}\left(z^{3}-3 z\right) /\left(24 \sigma^{4}\right)$ and

$$
G_{U, n}^{*}(z)=\Phi(z)-\frac{1}{n} \phi(z) P_{22}(z) .
$$

The coefficients of (5.1) are

and

$$
A_{1}=A_{3}=0
$$

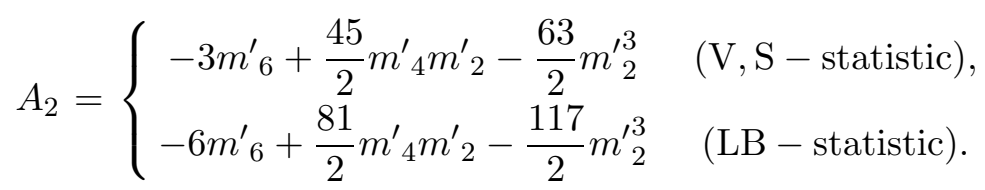


On the other hand, we have $G_{n}^{\prime \prime}(z)=G_{U, n}^{\prime}(z)=\Phi(z)$ and $H_{n}^{*}(z)=H_{n}(z)=$ $\Phi(z)$. Hence, we must consider the Edgeworth expansion of higher order to see the effect of Studentizing. We shall consider this problem in a future paper.

(2) We consider the kernel $g\left(x_{1}, x_{2}, \ldots, x_{k}\right)=x_{1} x_{2} \cdots x_{k}(k \geq 3)$. This kernel yields the estimable parameter $\theta(F)=\mu^{k}$, where $\mu$ is the mean of the distribution $F$. We assume that the distribution $F$ is symmetric about the mean $\mu(>0)$ and $E X^{2 k}<\infty$. We denote the central moments about the mean by $m_{j}$ $(j=2,4)$. Furthermore, we assume that $F$ have a density and the characteristic function $\psi$ of $F(x-\mu)$ is decreasing on $[0, \infty)$. We can write $g^{(2)}\left(x_{1}, x_{2}\right)=$ $\mu^{k-2}\left(x_{1}-\mu\right)\left(x_{2}-\mu\right)$.

Hence, we have $E \exp (i t[\alpha(X)+s f(X)])=\psi\left(\mu^{(k-2) / 2}\left[k \mu^{k / 2}+s\right] t\right)$. We shall take a sufficiently large $|t|$ such that $|t|^{-\varepsilon}<\mu$ for any $\varepsilon>0$. By decreasing values of $\psi$, for $|s| \leq|t|^{-\varepsilon}$ we have

$$
\psi\left(\mu^{(k-2) / 2}\left[k \mu^{k / 2}+s\right] t\right) \leq \max \left\{\psi\left(\mu^{(k-2) / 2}\left[k \mu^{k / 2} \pm|t|^{-\varepsilon}\right] t\right)\right\} .
$$

Thus $\sup _{|s| \leq|t|-\varepsilon} \psi\left(\mu^{(k-2) / 2}\left[k \mu^{k / 2}+s\right] t\right)$ converges to zero as $|t| \rightarrow \infty$, since $F$ has the density. Hence, the condition (D) is satisfied.

Now, we have

$$
\begin{aligned}
\sigma_{1}^{2} & =\mu^{2(k-1)} m_{2}, \\
\sigma_{2}^{2} & =\mu^{2(k-2)}\left(m_{2}^{2}+2 \mu^{2} m_{2}\right), \\
\sigma^{2} & =k^{2} \mu^{2(k-1)} m_{2}, \\
a_{3} & =0, \\
a_{4} & =k^{4} \mu^{4(k-1)} m_{4}, \\
a^{\prime} & =\delta_{k} k(k-2) \mu^{2(k-2)} m_{2}^{2}, \\
b= & k^{3}(k-1) \mu^{3 k-4} m_{2}^{2}, \\
c= & k^{4}(k-1)(k-2) \mu^{2(2 k-3)} m_{2}^{3}, \\
\kappa_{3}= & 3 k^{3}(k-1) \mu^{3 k-4} m_{2}^{2}, \\
\kappa_{4}= & k^{4}\left\{\mu^{4(k-1)} m_{4}+4(k-1)(4 k-5) \mu^{2(2 k-3)} m_{2}^{3}-3 \mu^{4(k-1)} m_{2}^{2}\right. \\
& \left.\quad-12(k-1)^{2} \mu^{2(2 k-1)} m_{2}-36(k-1) \mu^{4 k}\right\}, \\
\mu_{k}= & \delta_{k} \mu^{k-2} m_{2} .
\end{aligned}
$$

The coefficients necessary for the Edgeworth expansion are calculated by using $\delta_{k}$ and the moments $\mu, m_{2}, m_{4}$. In this case,

$$
\begin{aligned}
P_{1}(z)= & \frac{(k-1) \sqrt{m_{2}}}{2 \mu}\left(z^{2}-1\right), \\
P_{22}(z)= & \frac{\mu^{2} m_{4}+4(k-1)(4 k-5) m_{2}^{3}-3 \mu^{2} m_{2}^{2}-12(k-1)^{2} \mu^{4} m_{2}-36(k-1) \mu^{6}}{24 \mu^{2} m_{2}^{2}} \\
& \times\left(z^{3}-3 z\right)+\frac{(k-1)^{2} m_{2}}{8 \mu^{2}}\left(z^{5}-10 z^{3}+15 z\right)
\end{aligned}
$$

and 


$$
G_{U, n}^{*}(z)=\Phi(z)-\left[\frac{1}{\sqrt{n}} \phi(z) P_{1}(z)+\frac{1}{n} \phi(z) P_{22}(z)\right] .
$$

The coefficients of the right-hand side of (5.1) are as follows.

$$
\begin{aligned}
& A_{1}= \begin{cases}\frac{k(k-1) \mu^{k-2} m_{2}}{2} & (\mathrm{~V}, \mathrm{~S}-\text { statistic }) \\
k(k-1) \mu^{k-2} m_{2} & (\mathrm{LB}-\text { statistic }) .\end{cases} \\
& A_{2}= \begin{cases}\frac{k^{2}(k-1)(7 k-11) \mu^{2(k-2)} m_{2}^{2}}{8} & (\mathrm{~V}, \mathrm{~S}-\text { statistic }) \\
\frac{k^{2}(k-1)(7 k-11) \mu^{2(k-2)} m_{2}^{2}}{4} & (\mathrm{LB}-\text { statistic }) .\end{cases} \\
& A_{3}= \begin{cases}\frac{3 k^{4}(k-1)^{2} \mu^{2(2 k-3)} m_{2}^{3}}{2} & (\mathrm{~V}, \mathrm{~S}-\text { statistic }) \\
3 k^{4}(k-1)^{2} \mu^{2(2 k-3)} m_{2}^{3} & (\mathrm{LB}-\text { statistic }) .\end{cases}
\end{aligned}
$$

On the other hand,

$$
\begin{aligned}
& G_{n}^{\prime \prime}(z)=\Phi(z)-\frac{1}{\sqrt{n}}\left[\frac{\delta_{k} \sqrt{m_{2}}}{k \mu}+\frac{(k-1) \sqrt{m_{2}}}{2 \mu}\left(z^{2}-1\right)\right] \phi(z), \\
& G_{n}^{\prime \prime}(z)-G_{U, n}^{\prime}(z)=-\frac{(k-1) \sqrt{m_{2}}}{2 \mu \sqrt{n}} \phi(z) \quad(\mathrm{V}, \mathrm{S}-\text { statistic })
\end{aligned}
$$

and

$$
G_{n}^{\prime \prime}(z)-G_{U, n}^{\prime}(z)=-\frac{(k-1) \sqrt{m_{2}}}{\mu \sqrt{n}} \phi(z) \quad(\text { LB }- \text { statistic }) .
$$

While, under the condition $E|X|^{8+\varepsilon}<\infty$ for $\varepsilon$,

and

$$
\begin{aligned}
& H_{n}(z)=\Phi(z)+\frac{(k-1) \sqrt{m_{2}}}{2 \mu \sqrt{n}}\left(z^{2}+1\right) \phi(z) \\
& H_{n}^{*}(z)-H_{n}(z)=-\frac{(k-1) \sqrt{m_{2}}}{2 \mu \sqrt{n}} \phi(z) \quad(\mathrm{V}, \mathrm{S}-\text { statistic })
\end{aligned}
$$

$$
H_{n}^{*}(z)-H_{n}(z)=-\frac{(k-1) \sqrt{m_{2}}}{\mu \sqrt{n}} \phi(z) \quad(\mathrm{LB}-\text { statistic }) .
$$

\section{Acknowledgements}

The authors would like to express their hearty thanks to the referees for their careful reading of the manuscript and the useful comments.

\section{REFERENCES}

Bickel, P. J., Götze, F. and van Zwet, Z. R. (1986). The Edgeworth expansion for U-statistics of degree two, Ann. Statist., 14, 1463-1484. 
Callaert, H., Janssen, P. and Veraverbeke, N. (1980). An Edgeworth expansion for U-statistics, Ann. Statist., 8, 299-312.

Lai, T. L. and Wang, J. Q. (1993). Edgeworth Expansions for symmetric statistics with applications to bootstrap methods, Statistica Sinica, 3, 517-542.

Lee, A. J. (1990). U-statistics, Marcel Dekker, New York.

Maesono, Y. (1987). Edgeworth expansion for one-sample U-statistics, Bull. Infor. Cyber., 22, $189-197$.

Maesono, Y. (1995). On the normal approximations of studentized U-statistics, J. Japan Statist. Soc., 25, No. 1, 19-33.

Maesono, Y. (1996). An Edgeworth expansion of a linear combination of U-statistics, J. Japan Statist. Soc., 26, No. 2, 189-207.

Maesono, Y. and Yamato, H. (1994). U-statistics and related topics, Sugaku Expositions, 7, $43-58$.

Nomachi, T. and Yamato, H. (2001). Asymptotic comparisons of U-statistics, V-statistics and limits of Bayes estimates by deficiencies, J. Japan Statist. Soc., 31, 85-98.

Nomachi, T., Kondo, M. and Yamato, H. (2002). Higher order efficiency of linear combinations of U-statistics as estimators of estimable parameters, Scientiae Mathematicae Japonicae, 56, 95-106.

Sen, P. K. (1977). Some invariance principles relating to jacknifing and their role in sequential analysis, Ann. Statist., 5, 316-329.

Serfling, R. S. (1980). Approximation Theorems of Mathematical Statistics, Wiley, New York.

Shorack, G. R. (2000). Probability for Statisticians, Springer, New York.

Takahashi, H. (1988). A note on Edgeworth expansions for the von Mises Functionals, $J$. Multivariate Analysis, 24, 56-65.

Toda, K. and Yamato, H. (2001). Berry-Esseen bounds for some statistics including LB-statistic and V-statistic, J. Japan Statist. Soc., 31, No. 2, 225-237.

Yamato, H. (1977a). Relations between limiting Bayes estimates and the U-statistics for estimable parameters of degrees 2 and 3, Commun. Statist.-Theor. Meth., Series A, 6, 55-66.

Yamato, H. (1977b). Relations between limiting Bayes estimates ans U-statistics for estimable parameter, J. Japan Statist. Soc., 7, 57-66. 